\title{
Commutators of log-Dini-type parametric Marcinkiewicz operators on non-homogeneous metric measure spaces
}

Tao Xiangxing ${ }^{1}$ and Zhang Qiange ${ }^{1 *}$ (D)

"Correspondence:

qgzhang02@163.com

1 Department of Mathematics,

Zhejiang University of Science and

Technology, Hangzhou, 310023, P.R.

China

\begin{abstract}
Let $(\mathcal{X}, d, \mu)$ be a non-homogeneous metric measure space, which satisfies the geometrically doubling condition and the upper doubling condition. In this paper, the authors prove the boundedness in $L^{p}(\mu)$ of $m$ th-order commutators $\mathcal{M}_{b, m}^{\rho}$ generated by the Log-Dini-type parametric Marcinkiewicz integral operators with RBMO functions on $(\mathcal{X}, d, \mu)$. In addition, the boundedness of the $m$ th-order commutators $\mathcal{M}_{b, m}^{\rho}$ on Morrey spaces $M_{p}^{q}(\mu), 1<p \leq q<\infty$, is also obtained for the parameter $0<\rho<\infty$.
\end{abstract}

MSC: Primary 42B20; secondary 42B25

Keywords: Log-Dini-type parametric Marcinkiewicz operator; $\operatorname{RBMO}(\mu)$; Commutator; Non-homogeneous metric measure space

\section{Introduction}

Marcinkiewicz integral operators and their commutators play a very important role in harmonic analysis. Therefore, many authors have focused on studying the operators and their commutators. In 1960, Hörmander [1] introduced the parametric Marcinkiewicz integral, defined by,

$$
\mu_{\Omega}^{\rho}(f)(x)=\left(\int_{0}^{\infty}\left|\frac{1}{t^{\rho}} \int_{|x-y|<t} \frac{\Omega(x-y)}{|x-y|^{d-\rho}} f(y) d y\right|^{2} \frac{d t}{t}\right)^{\frac{1}{2}}
$$

where $\rho \in(0, \infty)$. Let $\Omega$ be homogeneous of degree zero in $R^{d}$ for $d \geq 2$, integrable and have mean value zero on the unit sphere $S^{d-1}$. Hörmander [1] proved that, if $\Omega \in \operatorname{Lip}_{\alpha}\left(S^{d-1}\right)$ for some $\alpha \in(0,1]$, then $\mu_{\Omega}^{\rho}$ is bounded on $L^{p}\left(R^{d}\right)$ for $p \in(1, \infty)$. In 2001, Fan [2] obtained the boundedness of $\mu_{\Omega}^{\rho}$ from $L^{1}\left(R^{d}\right)$ to $L^{1, \infty}\left(R^{d}\right)$ when $\Omega \in L(\log L)\left(S^{d-1}\right)$.

If $\rho=1$ in (1.1), then it is the higher-dimensional Marcinkiewicz integral first introduced by Stein [3] in 1958, denoted $\mu_{\Omega}$. Stein [3] proved that $\mu_{\Omega}$ is bounded on $L^{p}\left(R^{d}\right)$ for any $1<p \leq 2$, and is also bounded from $L^{1}\left(R^{d}\right)$ to $L^{1, \infty}\left(R^{d}\right)$. In 1990, Torchinsky and Wang [4] first introduced the commutator $\mu_{\Omega, b}$ generated by $\mu_{\Omega}$ and BMO function $b$, defined as

(c) The Author(s) 2021. This article is licensed under a Creative Commons Attribution 4.0 International License, which permits use, sharing, adaptation, distribution and reproduction in any medium or format, as long as you give appropriate credit to the original author(s) and the source, provide a link to the Creative Commons licence, and indicate if changes were made. The images or other third party material in this article are included in the article's Creative Commons licence, unless indicated otherwise in a credit line to the material. If material is not included in the article's Creative Commons licence and your intended use is not permitted by statutory regulation or exceeds the permitted use, you will need to obtain permission directly from the copyright holder. To view a copy of this licence, visit http://creativecommons.org/licenses/by/4.0/. 
follows:

$$
\mu_{\Omega, b}(f)(x)=b(x) \mu_{\Omega}(f)(x)-\mu_{\Omega}(b f)(x), \quad x \in R^{d},
$$

and established its $L^{p}\left(R^{d}\right)$ boundedness for $p \in(1, \infty)$. In 2002, Salman [5] reduced the condition of the kernel function to $\Omega \in L(\log \mathrm{L})^{\frac{1}{2}}\left(S^{d-1}\right)$, and proved that $\mu_{\Omega}$ is bounded on $L^{p}\left(R^{d}\right)$ for $p \in(1, \infty)$.

Recently, Gürbüz considered the boundedness of Marcinkiewicz integral operator with rough kernel associated with the Schrödinger operator and their commutators [6-8]. Gürbüz also proved some relevant conclusions about Marcinkiewicz operators, one may refer to [9-12]. In addition, Tao proved the boundedness of Marcinkiewicz integral operator with rough kernel [13-15]

In this paper, we will discuss the boundedness of commutators of the parametric Marcinkiewicz integral on the non-homogeneous metric space. Let $(\mathcal{X}, d)$ be a metric space, and let $\mu$ be a positive Borel measure on $\mathcal{X}$ that satisfies the following growth condition: for all $x \in \mathcal{X}, r>0$,

$$
\mu(B(x, r)) \leq C_{0} r^{n}
$$

where $C_{0}>0$ and $B(x, r):=\{y \in \mathcal{X}: d(x, y)<r\}$.

It is well known that the analysis on $(\mathcal{X}, d, \mu)$ played key roles in many fields, for example, in solving Painlevé's problem [16]. In 2010, Hytönen [17] introduced a non-homogeneous metric measure space, of which the measure satisfies the geometrically doubling condition and the upper doubling condition. From then on, many researchers considered singular integral operators on $(\mathcal{X}, d, \mu)$; see [18-20] for example. The purpose of this article is to consider the boundedness of the commutators generated by the Log-Dini-type parametric Marcinkiewicz integral with RBMO functions on $(\mathcal{X}, d, \mu)$. Before stating our results, we recall some notions of geometrically doubling and upper doubling measure [17].

Definition 1.1 ([17]) Let $(\mathcal{X}, d)$ is a metric space; if there exists some $N_{0} \in N$, and for any $x \in \mathcal{X}, r>0$, such that any ball $B(x, r) \subset \mathcal{X}$ can be covered by at most $N_{0}$ balls $B\left(x_{i}, \frac{r}{2}\right)$, we say $(\mathcal{X}, d)$ satisfies the geometrically doubling condition.

Definition $1.2([17])$ Let $(\mathcal{X}, d, \mu)$ is a metric measure space, if $\mu$ is a Borel measure on $\mathcal{X}$ and there exist a dominating function $\lambda(x, r): \mathcal{X} \times R_{+} \rightarrow R_{+}$and a constant $C_{\lambda}>0$ such that $r \rightarrow \lambda(x, r)$ is increasing and

$$
\mu(B(x, r)) \leq \lambda(x, r) \leq C_{\lambda} \lambda\left(x, \frac{r}{2}\right)
$$

for all $x \in \mathcal{X}, r>0$, then we say $\mu$ is an upper doubling measure.

We also need to recall other notions $[17,21]$.

Definition 1.3 For $\alpha, \beta \in(1, \infty)$, a ball $B \subset \mathcal{X}$ is called $(\alpha, \beta)$ doubling if

$$
\mu(\alpha B) \leq \beta \mu(B)
$$


One can see from Lemma 3.2 of [17] that, if $\mu$ is upper doubling, for any $\alpha, \beta \in(1, \infty)$ and $\beta>C_{\lambda}^{\log _{2} a}=: \alpha^{\nu}$, then for every ball $B \subset \mathcal{X}$ there exists $j \in n$, such that $\alpha^{j} B$ is $(\alpha, \beta)$ doubling ball. Moreover, we see from Lemma 3.3 of [17] that, if $(\mathcal{X}, d)$ is geometrically doubling, there exists $n_{0}:=\log _{2} N_{0}$, such that $\beta>\alpha^{n_{0}}$, if $\mu$ is a Borel measure on $\mathcal{X}$ which is finite on bounded sets, then, for $\mu$-a.e. $x \in \mathcal{X}$, there exist arbitrarily small $(\alpha, \beta)$ doubling balls centred at $x$. Moreover, for any preassigned $r>0$, their radius can be chosen to be of the form $\alpha^{j} r, j \in n$. Throughout this paper, fix $\tau \geq 1$, B is a $\left(30 \tau, \beta_{30 \tau}\right)$ doubling ball and

$$
\beta_{30 \tau}>\max \left\{(30 \tau)^{3 n}, c_{\lambda}^{3 \log _{2}(30 \tau)}\right\}
$$

For any $\tau \geq 1, B \subset \mathcal{X}, \tilde{B}$ denotes the smallest $\left(30 \tau, \beta_{30 \tau}\right)$ doubling ball of the form $(30 \tau)^{j} B$.

As in [7], for any two balls $B \subset S, r_{B}$ and $r_{S}$ denote the radius of the ball $\mathrm{B}$ and $\mathrm{S}$, respectively. And $x_{B}$ denotes the center of the ball B. We define $K_{B, S}$ and $\tilde{K}_{B, S}$ as follows:

$$
K_{B, S}:=1+\int_{r_{B} \leq d\left(x, x_{B}\right) \leq r_{S}} \frac{1}{\lambda\left(x_{B}, d\left(x, x_{B}\right)\right.} d \mu(x) .
$$

Let $N_{B, S}$ be the smallest integer satisfying $6^{N_{B, S}} r_{B} \geq r_{S}$, we define

$$
\tilde{K}_{B, S}:=1+\sum_{k=1}^{N_{B, S}} \frac{\mu\left(6^{k} B\right)}{\lambda\left(x_{B}, 6^{k} r_{B}\right)} .
$$

In the case that $\lambda(x, a r)=a^{m} \lambda(x, r)$ for all $x \in \mathcal{X}, a, r>0$, it is easy to show that $K_{B, S} \simeq \tilde{K}_{B, S}$. Nevertheless, in general, we only have $K_{B, S} \leq C \tilde{K}_{B, S}$.

Finally, we recall the definition of Morrey space [22] on $(\mathcal{X}, d, \mu)$.

Definition 1.4 Let $\kappa>1$ and $1 \leq p \leq q<\infty$, the definition of Morrey space are as follows:

$$
M_{p}^{q}(\kappa, \mu)=\left\{f \in L_{\mathrm{loc}}^{p}:\|f\|_{M_{p}^{q}(\kappa, \mu)}<\infty\right\}
$$

where

$$
\|f\|_{M_{p}^{q}(\kappa, \mu)}=\sup _{B} \mu(\kappa B)^{\frac{1}{q}-\frac{1}{p}}\left(\int_{B}|f|^{p} d \mu\right)^{\frac{1}{p}}
$$

We remark that, for any $\kappa_{1}, \kappa_{2}>1, M_{p}^{q}\left(\kappa_{1}, \mu\right)=M_{p}^{q}\left(\kappa_{2}, \mu\right)$ (see [23]). Particularly, if $\mu$ is a doubling measure, then $M_{p}^{q}(\kappa, \mu)=M_{p}^{q}(1, \mu)$ for any $\kappa>0$, and denote it by $M_{p}^{q}(\mu)$ for brevity. Moreover, it is easily to see that the space $M_{p}^{q}(\mu)$ becomes the classical Morrey space whenever $d \mu=d x$.

Next, we introduce the conditions of kernel discussed in this article.

Definition 1.5 Let $\omega:[0, \infty) \rightarrow[0, \infty)$ be non-decreasing function that satisfies

$$
\int_{0}^{1} \frac{\omega(t)}{t}|\log t| d t<\infty
$$


Let $K(x, y) \in L_{\text {loc }}^{1}\left((\mathcal{X})^{2} \backslash\{(x, y): x=y\}\right)$, we say $K(x, y)$ is the parametric Marcinkiewicz kernel of Log-Dini type, if there exists $C>0$ such that the following size estimate and smoothness estimates hold:

(i) For $x, y \in \mathcal{X}$ with $x \neq y$,

$$
|K(x, y)| \leq C \frac{d(x, y)}{\lambda(x, d(x, y))}
$$

(ii) For $x, x^{\prime}, y \in \mathcal{X}$ and if $2 d\left(x, x^{\prime}\right) \leq d(x, y)$,

$$
\left|K(x, y)-K\left(x^{\prime}, y\right)\right| \leq C \frac{d(x, y)}{\lambda(x, d(x, y))} \omega\left(\frac{d\left(x, x^{\prime}\right)}{d(x, y)}\right)
$$

(iii) For $x, y^{\prime}, y \in \mathcal{X}$ and if $2 d\left(y, y^{\prime}\right) \leq d(x, y)$,

$$
\left|K(x, y)-K\left(x, y^{\prime}\right)\right| \leq C \frac{d(x, y)}{\lambda(x, d(x, y))} \omega\left(\frac{d\left(y, y^{\prime}\right)}{d(x, y)}\right) .
$$

The parametric Marcinkiewicz integral $\mathcal{M}^{\rho}$ with Log-Dini-type kernel $K(x, y)$ satisfying (1.9), (1.10) and (1.11) is then defined, initially for $f \in L^{\infty}$ with compact support, by

$$
\mathcal{M}^{\rho}(f)(x)=\left(\int_{0}^{\infty}\left|\frac{1}{t^{\rho}} \int_{B(x, t)} \frac{K(x, y)}{|d(x, y)|^{1-\rho}} f(y) d \mu(y)\right|^{2} \frac{d t}{t}\right)^{\frac{1}{2}} .
$$

In case $\rho=1, \mathcal{M}^{\rho}$, denoted by $\mathcal{M}$, is just the Marcinkiewicz integral operator on $(\mathcal{X}, d, \mu)$ with Log-Dini-type kernel.

In 2014, Lin and Yang [24] proved that $\mathcal{M}$ is bounded on $L^{p}(\mu)$ if and only if $\mathcal{M}$ is bounded from $L^{1}(\mu)$ to $L^{1, \infty}(\mu)$, if the kernel $K(x, y)$ satisfies (1.9) and for all $x, y, y^{\prime} \in \mathcal{X}$

$$
\int_{d(x, y) \geq 2 d\left(y, y^{\prime}\right)}\left[\left|K(x, y)-K\left(x, y^{\prime}\right)\right|+\left|K(y, x)-K\left(y^{\prime}, x\right)\right|\right] \frac{d \mu(x)}{d(x, y)} \leq C .
$$

In 2016, Fu and Lin [25] proved that when the kernel $K(x, y)$ satisfies (1.9) and (1.13), if $\mathcal{M}^{\rho}$ is bounded on $L^{p_{0}}(\mu)$ with some $1<p_{0}<\infty$ then $\mathcal{M}^{\rho}$ is bounded from $L^{1}(\mu)$ to $L^{1, \infty}(\mu)$.

Given $b \in \operatorname{RBMO}(\mu)$, the commutators $\mathcal{M}_{b}^{\rho}$ generated by $\mathcal{M}^{\rho}$ with RBMO function $b$ is defined by

$$
\mathcal{M}_{b}^{\rho}(f)(x)=\left(\int_{0}^{\infty}\left|\frac{1}{t^{\rho}} \int_{B(x, t)} \frac{K(x, y)}{|d(x, y)|^{1-\rho}}[b(x)-b(y)] f(y) d \mu(y)\right|^{2} \frac{d t}{t}\right)^{\frac{1}{2}} .
$$

In general, for all $m \in \mathrm{N}$, the $m$ th-order commutators $\mathcal{M}_{b, m}^{\rho}$ is defined by

$$
\mathcal{M}_{b, m}^{\rho}(f)(x)=\left(\int_{0}^{\infty}\left|\frac{1}{t^{\rho}} \int_{B(x, t)} \frac{K(x, y)}{|d(x, y)|^{1-\rho}}[b(x)-b(y)]^{m} f(y) d \mu(y)\right|^{2} \frac{d t}{t}\right)^{\frac{1}{2}} .
$$

In 2015, Zhou [26] showed that the commutator $\mathcal{M}_{b}$ is bounded on $L^{p}(\mu)$, if $\mathcal{M}$ is bounded on $L^{2}(\mu)$, and the kernel $K(x, y)$ satisfies (1.9) and the following Hörmander type 
condition:

$$
\begin{aligned}
& \sup _{\substack{r>0 \\
d\left(y, y^{\prime}\right) \leq r}} \sum_{i=1}^{\infty} i \int_{6^{i} r<d(x, y) \leq 6^{i+1} r}\left[\left|K(x, y)-K\left(x, y^{\prime}\right)\right|\right. \\
& \left.+\left|K(y, x)-K\left(y^{\prime}, x\right)\right|\right] \frac{1}{d(x, y)} d \mu(x) \leq C .
\end{aligned}
$$

In 2019, Tao [27] proved that, if the kernel satisfies (1.9) and (1.16), then $\mathcal{M}_{b}$ is bounded on $M_{p}^{q}(\mu)$. In fact, we can see that (1.16) is stronger than (1.13).

In case $\mathcal{X}=R^{d}$, the non-homogeneous Euclidean space, then for the kernel $K(x, y)$ in the Marcinkiewicz integral it can be assumed that $K(x, y) \in L_{\mathrm{loc}}^{1}\left(R^{d} \times R^{d} \backslash\{(x, y): x=y\}\right)$ satisfies the following conditions with a constant $C>0$ :

$$
|K(x, y)| \leq C|x-y|^{-(d-1)}
$$

and

$$
\int_{|x-y| \geq 2\left|y-y^{\prime}\right|}\left[\left|K(x, y)-K\left(x, y^{\prime}\right)\right|+\left|K(y, x)-K\left(y^{\prime}, x\right)\right|\right] \frac{1}{|x-y|} d \mu(x) \leq C .
$$

for all $x, y, y^{\prime} \in R^{d}$ with $x \neq y$. And the Marcinkiewicz integral $\mathcal{M}$ is defined by

$$
\mathcal{M}(f)(x)=\left(\int_{0}^{\infty}\left|\frac{1}{t} \int_{|x-y|<t} K(x, y) f(y) d \mu(y)\right|^{2} \frac{d t}{t}\right)^{\frac{1}{2}} .
$$

In 2007, $\mathrm{Hu}$ [28] obtained $\mathcal{M}$ is bounded on $L^{p}(\mu), 1<p<\infty$, and is bounded from $L^{1}(\mu)$ to $L^{1, \infty}(\mu)$. Later, Zhang [29] proved $\mathcal{M}$ is bounded on $M_{p}^{q}(\mu)$.

For $m \in N$ and $b \in \mathrm{RBMO}$, the $m$ th-order commutator for Marcinkiewicz integral is denoted by

$$
\mathcal{M}_{b, m}(f)(x)=\left(\int_{0}^{\infty}\left|\frac{1}{t} \int_{|x-y| \leq t} K(x, y)[b(x)-b(y)]^{m} f(y) d \mu(y)\right|^{2} \frac{d t}{t}\right)^{\frac{1}{2}} .
$$

In 2007, $\mathrm{Hu}$ [28] proved that $\mathcal{M}_{b, m}$ is bounded on $L^{p}(\mu)$ if the kernel $K(x, y)$ satisfies (1.17) and the following condition:

$$
\begin{aligned}
& \sup _{\substack{r>0, y, y^{\prime} \in R^{n} \\
\left|y-y^{\prime}\right| \leq r}} \sum_{l=1}^{\infty} l^{m} \int_{2^{l_{r}<|x-y| \leq 2^{l+1} r}}\left[\left|K(x, y)-K\left(x, y^{\prime}\right)\right|\right. \\
& \left.+\left|K(y, x)-K\left(y^{\prime}, x\right)\right|\right] \frac{1}{|x-y|} d \mu(x) \leq C .
\end{aligned}
$$

It is easy to see that (1.21) is stronger than (1.18). In 2010, Zhang [29] proved that $\mathcal{M}_{b}$ is bounded on $M_{p}^{q}(\mu)$ under the same assumptions.

Now we turn to stating the main results of this paper. 
Theorem 1.1 Let $K$ satisfy (1.9), (1.10), and (1.11). $\mathcal{M}^{\rho}, \mathcal{M}_{b}^{\rho}$ be as in (1.12) and (1.14), respectively. Suppose that $\mathcal{M}^{\rho}$ is bounded on $L^{2}(\mu), b \in \operatorname{RBMO}(\mu), 0<\rho<\infty$. If $\omega$ satisfies

$$
\int_{0}^{1} \frac{\omega(t)}{t}|\log t| d t<\infty
$$

then, for all $f \in L^{p}(\mu), 1<p<\infty$, there exists a constant $C>0$ such that

$$
\left\|\mathcal{M}_{b}^{\rho}(f)\right\|_{L^{p}(\mu)} \leq C\|b\|_{\operatorname{RBMO}(\mu)}\|f\|_{L^{p}(\mu)} .
$$

In fact we will prove the $L^{p}(\mu)$ boundedness for a more general $m$ th-order commutator for the parametric Marcinkiewicz integral.

Theorem 1.2 Under the same conditions of Theorem 1.1 and $\mathcal{M}_{b, m}^{\rho}$ be as in (1.15). If $\omega$ satisfies the following condition:

$$
\int_{0}^{1} \frac{\omega(t)}{t}|\log t|^{m} d t<\infty
$$

then for all $f \in L^{p}(\mu), 1<p<\infty$, there exists a constant $C>0$ such that

$$
\left\|\mathcal{M}_{b, m}^{\rho}(f)\right\|_{L^{p}(\mu)} \leq C\|b\|_{\mathrm{RBMO}(\mu)}^{m}\|f\|_{L^{p}(\mu)} .
$$

Theorem 1.1 is the special case of Theorem 1.2 in which one can take $m=1$. We will prove Theorem 1.2 in Sect. 2.

Moreover, we will establish the boundedness of $\mathcal{M}_{b, m}^{\rho}$ on the Morrey space.

Theorem 1.3 Assume the same conditions of Theorem 1.1 and $\mathcal{M}_{b, m}^{\rho}$ as in (1.15). If $\omega$ satisfies (1.24), then there exists a constant $C>0$, for all $f \in M_{p}^{q}(\mu), 1<p \leq q<\infty$, such that

$$
\left\|\mathcal{M}_{b, m}^{\rho}(f)\right\|_{M_{p}^{q}(\mu)} \leq C\|b\|_{\mathrm{RBMO}(\mu)}^{m}\|f\|_{M_{p}^{q}(\mu)}
$$

By checking the proofs of Theorem 1.2 and Theorem 1.3, we can obtain the following two corollaries, which extend the results in [26] and [27].

Corollary 1.4 Let the kernel $K(x, y)$ satisfy (1.9) and (1.16), $\mathcal{M}^{\rho}$ and $\mathcal{M}_{b, m}^{\rho}$ be as in (1.12) and (1.15), respectively. Suppose that $\mathcal{M}^{\rho}$ is bounded on $L^{2}(\mu), b \in \operatorname{RBMO}(\mu), 0<\rho<\infty$. If $\omega$ satisfies (1.24), then there exists a constant $C>0$, for all $f \in L^{p}(\mu), 1<p<\infty$, such that

$$
\left\|\mathcal{M}_{b, m}^{\rho}(f)\right\|_{L^{p}(\mu)} \leq C\|b\|_{\mathrm{RBMO}(\mu)}^{m}\|f\|_{L^{p}(\mu)}
$$

Corollary 1.5 Under the same conditions of Corollary 1.4, there exists a constant $C>0$, for all $f \in M_{p}^{q}(\mu), 1<p \leq q<\infty$, such that

$$
\left\|\mathcal{M}_{b, m}^{\rho}(f)\right\|_{M_{p}^{q}(\mu)} \leq C\|b\|_{\mathrm{RBMO}(\mu)}^{m}\|f\|_{M_{p}^{q}(\mu)} .
$$


Remark 1.6 If $\rho=1, m=1$ on Corollary 1.4, which is Theorem 1.10 of [26]; if $\rho=1, m=1$ on Corollary 1.5, which is Theorem 1.8 of [27], so our results contain their conclusions.

Throughout this paper, $d$ is the dimension of space; $C$ denotes a positive constant that is independent of the parameters, furthermore, it value may differ from line to line; $x_{B}$ denotes the center of the ball $B, r_{B}$ denotes the radius of the ball $B$; for any $p \in(1, \infty)$, we denote by $p^{\prime}=\frac{p}{p-1}$ its conjugate index; $m_{B}(b)$ is the mean value of $B$ on $B$, namely $m_{B}(b)=\frac{1}{\mu(B)} \int_{B} b(x) d \mu(x)$.

\section{Proof of Theorem 1.2}

We first recall the definition of a sharp maximal operator $M^{\sharp} f(x)[21]$ over $(\mathcal{X}, d, \mu)$. For any $f \in L_{\text {loc }}^{1}(\mu)$,

$$
M^{\sharp} f(x)=\sup _{x \in B} \frac{1}{\mu(6 B)} \int_{B}\left|f-m_{\tilde{B}}(f)\right| d \mu+\sup _{(B, S) \in \Delta_{x}} \frac{\left|m_{B}(f)-m_{S}(f)\right|}{K_{B, S}},
$$

here $\Delta_{x}=\{(B, S): x \in B \subset S, B$, Sare doubling balls $\}$. As usual, we let $M_{\delta}^{\sharp}(f)(x)=$ $\left[M^{\sharp}\left(|f(x)|^{\delta}\right)\right]^{\frac{1}{\delta}}$

We will use the following lemma about sharp maximal function on $(\mathcal{X}, d, \mu)$ proved by $\mathrm{Fu}[18]$.

Lemma 2.1 (i) Let $p>1, s \in[1, p), \zeta \in[5, \infty)$. For all $f \in L_{\mathrm{loc}}^{1}(\mu)$ and $x \in \mathcal{X}$,

$$
M_{s, \zeta} f(x)=\sup _{x \in B}\left(\frac{1}{\mu(\zeta B)} \int_{B}|f(y)|^{s} d \mu(y)\right)^{\frac{1}{s}}
$$

is bounded on $L^{p}(\mu)$ and also bounded from $L^{1}(\mu)$ to $L^{1, \infty}(\mu)$. If $s=1$, then $M_{s, \zeta} f=M_{(\zeta)} f$.

(ii) For any $\delta \in(0,1)$ and for $f \in L_{\mathrm{loc}}^{1}(\mu)$, define

$$
N_{\delta} f(x):=\sup _{x \in B: \text { doubling }}\left(\frac{1}{\mu(B)} \int_{B}|f(y)|^{\delta} d \mu(y)\right)^{\frac{1}{\delta}},
$$

then, for $\mu$-almost every $x \in \mathcal{X}$,

$$
|f(x)| \leq N_{\delta} f(x)
$$

According to Theorem 4.2 in [21], we can easily get the following lemma.

Lemma 2.2 Let $f \in L_{\text {loc }}^{1}(\mu)$ satisfy $\int_{\mathcal{X}} f d \mu=0$ when $\|\mu\|:=\mu(\mathcal{X})<\infty$. Assume that $\inf \left\{1, N_{\delta} f\right\} \in L^{p}(\mu)$, for any $p \in(1, \infty), \delta \in(0,1)$, then there exists a constant $C>0$,

$$
\left\|N_{\delta} f\right\|_{L^{p}(\mu)} \leq C\left\|M_{\delta}^{\sharp}(f)\right\|_{L^{p}(\mu)} .
$$

The next two lemmas can be found in [30].

Lemma 2.3 Let $\varrho>1$, for $b \in L_{\text {loc }}^{1}(\mu)$. The following statements are equivalent:

(i) $b \in \operatorname{RBMO}(\mu)$. 
(ii) There exists a constant $C>0$, such that, for all balls $B$,

$$
\frac{1}{\mu(\varrho B)} \int_{B}\left|b(x)-m_{\tilde{B}}(b)\right| d \mu(x) \leq C,
$$

and for all $\left(30 \tau, \beta_{30 \tau}\right)$ doubling balls $B \subset S$,

$$
\left|m_{B}(b)-m_{S}(b)\right| \leq C K_{B, S}
$$

where $m_{B}(b)=\frac{1}{\mu(B)} \int_{B} b(x) d \mu(x)$. Furthermore, the infimum of all positive constants $C$ satisfying (2.5) and (2.6) is an equivalent $R B M O$ norm of $b$, denoted by $\|b\|_{\operatorname{RBMO}(\mu)}$.

Lemma 2.4 Let $\varrho>1, p \in[1, \infty)$, if $b \in \mathrm{RBMO}$, for any ball $B$, then there exists a constant $C>0$, we have

$$
\left(\frac{1}{\mu(\varrho B)} \int_{B}\left|b(x)-m_{\tilde{B}}(b)\right|^{p} d \mu(x)\right)^{\frac{1}{p}} \leq C\|b\|_{\operatorname{RBMO}(\mu)} .
$$

We need the following lemma about the boundedness of parametric Marcinkiewicz integral operators.

Lemma 2.5 Let kernel $K(x, y) \in L_{\text {loc }}^{1}\left((\mathcal{X})^{2} \backslash\{(x, y): x=y\}\right)$ satisfy (1.9), (1.10) and (1.11), $\mathcal{M}^{\rho}$ be as in (1.12), $0<\rho<\infty$. If $\mathcal{M}^{\rho}$ is bounded $L^{p_{0}}(\mu), 1<p_{0}<\infty$, then $\mathcal{M}^{\rho}$ is bounded from $L^{1}(\mu)$ to $L^{1, \infty}(\mu)$.

Proof In Theorem 2.1 of [25], the kernel function satisfies (1.9) and (1.13). It is easily to see that (1.13) is weaker than (1.10) and (1.11). So by similar argument as that in Theorem 2.1 of [25], we can prove the lemma. Hence, we omit the details.

To prove Theorem 1.2, we should first establish the following lemma.

Lemma 2.6 Let $K(x, y)$ satisfy (1.9), (1.10) and (1.11). Suppose $\mathcal{M}^{\rho}$ be as in (1.12) is bounded on $L^{2}(\mu), b \in \operatorname{RBMO}(\mu)$. If $0<\rho<\infty, \delta \in(0,1)$ and $\omega$ satisfies (1.24), then there exists a constant $C>0$, for all $f \in L^{p}(\mu)$, such that

$$
\begin{aligned}
M_{\delta}^{\sharp}\left[\mathcal{M}_{b, m}^{\rho}(f)(x)\right] \leq & C\left[\sum_{k=0}^{m-1}\|b\|_{\mathrm{RBMO}(\mu)}^{m-k} M_{\eta, 30 \tau}\left[\mathcal{M}_{b, k}^{\rho}(f)\right](x)\right. \\
& \left.+\|b\|_{\mathrm{RBMO}(\mu)}^{m} M_{p, 30 \tau}(f)(x)\right] .
\end{aligned}
$$

Here $\mathcal{M}_{b, 1}^{\rho}=\mathcal{M}_{b}^{\rho}$ and $\mathcal{M}_{b, 0}^{\rho}=\mathcal{M}^{\rho}$.

Proof Without loss of generality, we may assume that $\|b\|_{\operatorname{RBMO}(\mu)}=1$. In order to prove (2.8), it suffices to prove that, for all $x \in \mathcal{X}$ and balls $B \ni x$,

$$
\left[\frac{1}{\mu(30 \tau B)} \int_{B}\left|\mathcal{M}_{b, m}^{\rho}(f)(y)-h_{B}\right|^{\delta} d \mu(y)\right]^{\frac{1}{\delta}}
$$




$$
\leq C\left[\sum_{k=0}^{m-1} M_{\eta, 30 \tau}\left[\mathcal{M}_{b, k}^{\rho}(f)\right](x)+M_{p, 30 \tau}(f)(x)\right],
$$

and for all balls $B \subset S, S$ is a $\left(30 \tau, \beta_{30 \tau}\right)$ doubling ball,

$$
\begin{aligned}
& \left|h_{B}-h_{S}\right| \\
& \quad \leq C\left[K_{B, S}\right]^{m}\left[\sum_{k=0}^{m-1} M_{\eta, 30 \tau}\left[\mathcal{M}_{b, k}^{\rho}(f)\right](x)+M_{p, 30 \tau}(f)(x)\right],
\end{aligned}
$$

where

$$
\begin{aligned}
h_{B} & :=m_{B}\left[\mathcal{M}^{\rho}\left(\left[b-m_{\tilde{B}}(b)\right]^{m} f \chi \mathcal{X} \backslash 6 B\right)\right], \\
h_{S} & :=m_{S}\left[\mathcal{M}^{\rho}\left(\left[b-m_{\tilde{S}}(b)\right]^{m} f \chi \mathcal{X} \backslash 6 S\right)\right] .
\end{aligned}
$$

Now we decompose the function $f$ into two parts, i.e., $f=f \chi_{6 B}+f \chi_{\mathcal{X} \backslash 6 B}:=f_{1}+f_{2}$. We can write

$$
\begin{aligned}
& {[b(y)-b(z)]^{m}} \\
& \quad=\left[m_{\tilde{B}}(b)-b(z)\right]^{m}-\sum_{k=0}^{m-1} C_{m}^{k}[b(y)-b(z)]^{k}\left[m_{\tilde{B}}(b)-b(y)\right]^{m-k} .
\end{aligned}
$$

Thus, we obtain

$$
\begin{aligned}
& \mathcal{M}_{b, m}^{\rho}(f)(y) \\
& \quad=\left(\int_{0}^{\infty}\left|\frac{1}{t^{\rho}} \int_{B(y, t)} \frac{K(y, z)}{|d(y, z)|^{1-\rho}}[(b(y)-b(z))]^{m} f(z) d \mu(z)\right|^{2} \frac{d t}{t}\right)^{\frac{1}{2}} \\
& \quad \leq \sum_{k=0}^{m-1} C_{m}^{k}\left|m_{\tilde{B}}(b)-b(y)\right|^{m-k} \mathcal{M}_{b, k}^{\rho}(f)(y)+\mathcal{M}^{\rho}\left(\left[b(\cdot)-m_{\tilde{B}}(b)\right]^{m} f\right)(y) .
\end{aligned}
$$

Since $0<\delta<1$,

$$
\begin{aligned}
& {\left[\frac{1}{\mu(30 \tau B)} \int_{B}\left|\mathcal{M}_{b, m}^{\rho}(f)(y)-h_{B}\right|^{\delta} d \mu(y)\right]^{\frac{1}{\delta}}} \\
& \leq\left[\frac{1}{\mu(30 \tau B)} \int_{B}\left|\sum_{k=0}^{m-1} C_{m}^{k}\right| m_{\tilde{B}}(b)-\left.\left.b(y)\right|^{m-k} \mathcal{M}_{b, k}^{\rho}(f)(y)\right|^{\delta} d \mu(y)\right]^{\frac{1}{\delta}} \\
& \quad+\left[\frac{1}{\mu(30 \tau B)} \int_{B}\left|\mathcal{M}^{\rho}\left(\left[b(\cdot)-m_{\tilde{B}}(b)\right]^{m} f_{1}\right)(y)\right|^{\delta} d \mu(y)\right]^{\frac{1}{\delta}} \\
& \quad+\left[\frac{1}{\mu(30 \tau B)} \int_{B}\left|\mathcal{M}^{\rho}\left(\left[b(\cdot)-m_{\tilde{B}}(b)\right]^{m} f_{2}\right)(y)-h_{B}\right|^{\delta} d \mu(y)\right]^{\frac{1}{\delta}} \\
& =: \\
& E_{1}+E_{2}+E_{3} .
\end{aligned}
$$


To estimate $E_{1}$, let $\gamma, \eta>1$, be such that

$$
\frac{1}{\gamma}+\frac{1}{\eta}=\frac{1}{\delta} .
$$

By Hölder's inequality and Lemma 2.4, we have

$$
\begin{aligned}
E_{1} \leq & \sum_{k=0}^{m-1} C_{m}^{k}\left[\frac{1}{\mu(30 \tau B)}\left(\int_{B}\left|\left[m_{\tilde{B}}(b)-b(y)\right]^{m-k}\right|^{\delta \cdot \frac{\gamma}{\delta}} d \mu(y)\right)^{\frac{\delta}{\gamma}}\right. \\
& \left.\times\left(\int_{B}\left|\mathcal{M}_{b, k}^{\rho}(f)(y)\right|^{\delta \cdot \frac{\eta}{\delta}} d \mu(y)\right)^{\frac{\delta}{\eta}}\right]^{\frac{1}{\delta}} \\
\leq & C \sum_{k=0}^{m-1}\|b\|_{\mathrm{RBMO}(\mu)}^{m-k}\left(\frac{1}{\mu(30 \tau B)}\left(\int_{B}\left|\mathcal{M}_{b, k}^{\rho}(f)(y)\right|^{\eta} d \mu(y)\right)^{\frac{1}{\eta}}\right) \\
\leq & C \sum_{k=0}^{m-1} M_{\eta, 30 \tau}\left(\mathcal{M}_{b, k}^{\rho}(f)\right)(x) .
\end{aligned}
$$

For $E_{2}$, by the Kolmogorov inequality, Lemma 2.5, Hölder's inequality and Lemma 2.4, we have

$$
\begin{aligned}
E_{2} & =\left[\frac{1}{\mu(30 \tau B)} \int_{B}\left|\mathcal{M}^{\rho}\left(\left[b(\cdot)-m_{\tilde{B}}(b)\right]^{m} f_{1}\right)(y)\right|^{\delta} d \mu(y)\right]^{\frac{1}{\delta}} \\
& \leq\left\|\mathcal{M}^{\rho}\left(\left[b(\cdot)-m_{\tilde{B}}(b)\right]^{m} f_{1}\right)(y)\right\|_{L^{1, \infty}\left(6 B, \frac{d \mu(y)}{\mu(30 \tau B)}\right)} \\
& \leq C \frac{1}{\mu(30 \tau B)} \int_{B}\left|b(y)-m_{\tilde{B}}(b)\right|^{m} \cdot\left|f_{1}(y)\right| d \mu(y) \\
& \leq C\left(\frac{1}{\mu(30 \tau B)} \int_{B}\left|b(y)-m_{\tilde{B}}(b)\right|^{m \cdot p^{\prime}} d \mu(y)\right)^{\frac{1}{p^{\prime}}}\left(\frac{1}{\mu(30 \tau B)} \int_{B}|f(y)|^{p} d \mu(y)\right)^{\frac{1}{p}} \\
& \leq C\|b\|_{\mathrm{RBMO}(\mu)}^{m} M_{p, 30 \tau}(f)(x) \\
& \leq C M_{p, 30 \tau}(f)(x) .
\end{aligned}
$$

As to the estimate $E_{3}$, we observe that

$$
\begin{aligned}
\mid \mathcal{M}^{\rho}( & {\left.\left[b(\cdot)-m_{\tilde{B}}(b)\right]^{m} f_{2}\right)(y)-h_{B} \mid } \\
= & \mid \frac{1}{\mu(B)} \int_{B}\left(\int_{0}^{\infty} \mid \int_{d(y, z)<t} \frac{K(y, z)}{|d(y, z)|^{1-\rho}}\right. \\
& \left.\times\left.\left[b(z)-m_{\tilde{B}}(b)\right]^{m} f_{2}(z) d \mu(z)\right|^{2} \frac{d t}{t^{2 \rho+1}}\right)^{\frac{1}{2}} d \mu(w) \\
& -\frac{1}{\mu(B)} \int_{B}\left(\int_{0}^{\infty} \mid \int_{d(w, z)<t} \frac{K(w, z)}{|d(w, z)|^{1-\rho}}\right. \\
& \left.\times\left.\left[b(z)-m_{\tilde{B}}(b)\right]^{m} f_{2}(z) d \mu(z)\right|^{2} \frac{d t}{t^{2 \rho+1}}\right)^{\frac{1}{2}} d \mu(w) \mid \\
= & \left|\frac{1}{\mu(B)} \int_{B}\right| \mathcal{M}^{\rho}\left(\left[b(\cdot)-m_{\tilde{B}}(b)\right]^{m} f_{2}\right)(y)-\mathcal{M}^{\rho}\left(\left[b(\cdot)-m_{\tilde{B}}(b)\right]^{m} f_{2}\right)(w)|d \mu(w)| .
\end{aligned}
$$


Hence

$$
\begin{aligned}
E_{3} \leq & {\left[\frac { 1 } { \mu ( 3 0 \tau B ) } \int _ { B } \left(\frac{1}{\mu(B)} \int_{B} \mid \mathcal{M}^{\rho}\left(\left[b(\cdot)-m_{\tilde{B}}(b)\right]^{m} f_{2}\right)(y)\right.\right.} \\
& \left.\left.-\mathcal{M}^{\rho}\left(\left[b(\cdot)-m_{\tilde{B}}(b)\right]^{m} f_{2}\right)(\omega) \mid d \mu(\omega)\right)^{\delta} d \mu(y)\right]^{\frac{1}{\delta}} .
\end{aligned}
$$

In fact, for $y, w \in B$, we observe that

$$
\begin{aligned}
& \left|\mathcal{M}^{\rho}\left(\left[b(\cdot)-m_{\tilde{B}}(b)\right]^{m} f_{2}\right)(y)-\mathcal{M}^{\rho}\left(\left[b(\cdot)-m_{\tilde{B}}(b)\right]^{m} f_{2}\right)(\omega)\right| \\
& \leq\left(\int_{0}^{\infty}\left|\int_{d(y, z)<t} \frac{K(y, z)}{|d(y, z)|^{1-\rho}}\left[b(z)-m_{\tilde{B}}(b)\right]^{m} f_{2}(z) d \mu(z)\right|^{2}\right. \\
& \left.-\left|\int_{d(w, z)<t} \frac{K(w, z)}{|d(w, z)|^{1-\rho}}\left[b(z)-m_{\tilde{B}}(b)\right]^{m} f_{2}(z) d \mu(z)\right|^{2} \frac{d t}{t^{2 \rho+1}}\right)^{\frac{1}{2}} \\
& \leq\left(\int_{0}^{\infty} \mid \int_{d(y, z)<t} \frac{K(y, z)}{|d(y, z)|^{1-\rho}}\left[b(z)-m_{\tilde{B}}(b)\right]^{m} f_{2}(z) d \mu(z)\right. \\
& \left.-\left.\int_{d(w, z)<t} \frac{K(w, z)}{|d(w, z)|^{1-\rho}}\left[b(z)-m_{\tilde{B}}(b)\right]^{m} f_{2}(z) d \mu(z)\right|^{2} \frac{d t}{t^{2 \rho+1}}\right)^{\frac{1}{2}} \\
& \leq\left(\int_{0}^{\infty}\left|\int_{d(y, z) \leq t \leq d(w, z)} \frac{K(y, z)}{|d(y, z)|^{1-\rho}}\left[b(z)-m_{\tilde{B}}(b)\right]^{m} f_{2}(z) d \mu(z)\right|^{2} \frac{d t}{t^{2 \rho+1}}\right)^{\frac{1}{2}} \\
& +\left(\int_{0}^{\infty}\left|\int_{d(w, z) \leq t \leq d(y, z)} \frac{K(w, z)}{|d(w, z)|^{1-\rho}}\left[b(z)-m_{\tilde{B}}(b)\right]^{m} f_{2}(z) d \mu(z)\right|^{2} \frac{d t}{t^{2 \rho+1}}\right)^{\frac{1}{2}} \\
& +\left(\int_{0}^{\infty} \mid \int_{\max \{d(y, z), d(w, z)\}<t}\left(\frac{K(y, z)}{|d(y, z)|^{1-\rho}}-\frac{K(w, z)}{|d(w, z)|^{1-\rho}}\right)\right. \\
& \left.\times\left.\left[b(z)-m_{\tilde{B}}(b)\right]^{m} f_{2}(z) d \mu(z)\right|^{2} \frac{d t}{t^{2 \rho+1}}\right)^{\frac{1}{2}} \\
& :=F_{1}+F_{2}+F_{3}
\end{aligned}
$$

In order to estimate $E_{3}$, it suffices to estimate $F_{1}, F_{2}$, and $F_{3}$. To estimate $F_{1}$, for all $y, w \in B$, we have $d(y, z) \sim d(w, z) \sim d\left(c_{B}, z\right)$. By the Minkowski inequality, (1.9), Hölder's inequality, and Lemma 2.4, we get

$$
\begin{aligned}
F_{1} & \leq \int_{\mathcal{X} \backslash 6 B} \frac{|K(y, z)|}{|d(y, z)|^{1-\rho}}\left|b(z)-m_{\tilde{B}}(b)\right|^{m}|f(z)|\left(\int_{d(y, z) \leq t \leq d(w, z)} \frac{d t}{t^{2 \rho+1}}\right)^{\frac{1}{2}} d \mu(z) \\
& \leq C \int_{\mathcal{X} \backslash 6 B} \frac{|d(y, z)|^{\rho}}{\lambda(y, d(y, z))}\left|b(z)-m_{\tilde{B}}(b)\right|^{m}|f(z)| \frac{1}{|d(y, z)|^{\rho}}\left(\frac{d(w, y)}{d(w, z)}\right)^{\frac{1}{2}} d \mu(z) \\
& \leq C \sum_{k=1}^{\infty} \int_{6^{k+1} B \backslash 6^{k} B}\left(\frac{r_{B}}{6^{k} r_{B}}\right)^{\frac{1}{2}} \frac{1}{\lambda\left(c_{B}, 6^{k} r_{B}\right)}\left|b(z)-m_{\tilde{B}}(b)\right|^{m}|f(z)| d \mu(z) \\
& \leq C \sum_{k=1}^{\infty}\left(\frac{1}{6^{\frac{k}{2}}} \frac{1}{\lambda\left(c_{B}, 6^{k} r_{B}\right)}\right)\left[\int_{6^{k+1} B}\left|b(z)-m_{6^{k+1} B}(b)\right|^{m}|f(z)| d \mu(z)\right.
\end{aligned}
$$




$$
\begin{aligned}
& \left.+\int_{6^{k+1} B}\left|m_{6^{k+1} B}(b)-m_{\tilde{B}}(b)\right|^{m}|f(z)| d \mu(z)\right] \\
\leq & C \sum_{k=1}^{\infty}\left(\frac{1}{6^{\frac{k}{2}}} \frac{1}{\lambda\left(c_{B}, 6^{k} r_{B}\right)}\right)\left(\int_{6^{k+1} B}|f(z)|^{p} d \mu(z)\right)^{\frac{1}{p}} \\
& \times\left[\left(\int_{6^{k+1} B}\left|b(z)-m_{6^{k+1} B}(b)\right|^{m \cdot p^{\prime}} d \mu(z)\right)^{\frac{1}{p^{\prime}}}\right. \\
& \left.+k^{m}\|b\|_{\mathrm{RBMO}(\mu)}^{m}\left[\mu\left(30 \tau \times 6^{k+1} B\right)\right]^{1-\frac{1}{p}}\right] \\
\leq & C\|b\|_{\mathrm{RBMO}(\mu)}^{m} M_{p, 30 \tau}(f)(x) \sum_{k=1}^{\infty}\left(\frac{k^{m}+1}{6^{\frac{k}{2}}} \frac{\mu\left(30 \tau \times 6^{k+1} B\right)}{\lambda\left(c_{B}, 6^{k} r_{B}\right)}\right) \\
\leq & C M_{p, 30 \tau}(f)(x) .
\end{aligned}
$$

We have used the following fact in the last inequality:

$$
\begin{aligned}
\frac{\mu\left(30 \tau \times 6^{k+1} B\right)}{\lambda\left(c_{B}, 6^{k} r_{B}\right)} & \leq \frac{\mu\left(6^{k+1} B\right)}{\lambda\left(c_{B}, 6^{k} r_{B}\right)} \\
& \leq \frac{\mu\left(6^{k+1} B\right)}{\mu\left(6^{k} B\right)} \cdot \frac{\mu\left(6^{k} B\right)}{\lambda\left(c_{B}, 6^{k} r_{B}\right)} \\
& \leq C .
\end{aligned}
$$

Similarly, we get

$$
F_{2} \leq C M_{p, 30 \tau}(f)(x)
$$

To estimate $F_{3}$, for all $y, w \in B$, we have $d(y, z) \sim d(w, z) \sim d\left(c_{B}, z\right)$, using the Minkowski inequality, we get

$$
\begin{aligned}
F_{3} \leq & \int_{\mathcal{X} \backslash 6 B}\left|\frac{K(y, z)}{|d(y, z)|^{1-\rho}}-\frac{K(w, z)}{|d(w, z)|^{1-\rho}}\right| \\
& \times\left|b(z)-m_{\tilde{B}}(b)\right|^{m}|f(z)|\left(\int_{d(y, z)}^{\infty} \frac{d t}{t^{2 \rho+1}}\right)^{\frac{1}{2}} d \mu(z) \\
\leq & \int_{\mathcal{X} \backslash 6 B}\left|\frac{K(y, z)}{|d(y, z)|^{1-\rho}}-\frac{K(w, z)}{|d(y, z)|^{1-\rho}}\right| \\
& \times\left|b(z)-m_{\tilde{B}}(b)\right|^{m}|f(z)|\left(\int_{d(y, z)}^{\infty} \frac{d t}{t^{2 \rho+1}}\right)^{\frac{1}{2}} d \mu(z) \\
& +\int_{\mathcal{X} \backslash 6 B}\left|\frac{K(w, z)}{|d(y, z)|^{1-\rho}}-\frac{K(w, z)}{|d(w, z)|^{1-\rho}}\right| \\
& \times\left|b(z)-m_{\tilde{B}}(b)\right|^{m}|f(z)|\left(\int_{d(y, z)}^{\infty} \frac{d t}{t^{2 \rho+1}}\right)^{\frac{1}{2}} d \mu(z) \\
:= & F_{31}+F_{32} .
\end{aligned}
$$


Next we estimate $F_{31}$ and $F_{32}$, respectively. For $F_{31}$, by (1.10), Hölder's inequality, Lemma 2.4 and (1.24), we have

$$
\begin{aligned}
F_{31} \leq & C \int_{\mathcal{X} \backslash 6 B}\left|\frac{d(y, z)}{\lambda(y, d(y, z))}\right| \omega\left(\frac{d(y, w)}{d(y, z)}\right) \frac{|f(z)|}{|d(y, z)|}\left|b(z)-m_{\tilde{B}}(b)\right|^{m} d \mu(z) \\
\leq & C \sum_{k=1}^{\infty} \int_{6^{k+1} B \backslash 6^{k} B} \omega\left(\frac{r_{B}}{6^{k} r_{B}}\right) \frac{1}{\lambda\left(c_{B}, 6^{k} r_{B}\right)}\left|b(z)-m_{\tilde{B}}(b)\right|^{m}|f(z)| d \mu(z) \\
\leq & C \sum_{k=1}^{\infty} \omega\left(6^{-k}\right) \frac{1}{\lambda\left(c_{B}, 6^{k} r_{B}\right)}\left[\int_{6^{k+1} B}\left|b(z)-m_{6^{k+1} B}(b)\right|^{m}|f(z)| d \mu(z)\right. \\
& \left.+\int_{6^{k+1} B}\left|m_{6^{k+1} B}(b)-m_{\tilde{B}}(b)\right|^{m}|f(z)| d \mu(z)\right] \\
\leq & C \sum_{k=1}^{\infty} \omega\left(6^{-k}\right) \frac{1}{\lambda\left(c_{B}, 6^{k} r_{B}\right)}\left(\int_{6^{k+1} B}|f(z)|^{p} d \mu(z)\right)^{\frac{1}{p}} \\
& \times\left[\left(\int_{6^{k+1} B}\left|b(z)-m_{6^{k+1} B}(b)\right|^{m \cdot p^{\prime}} d \mu(z)\right)^{\frac{1}{p^{\prime}}}\right. \\
& \left.+C m_{6^{k+1} B}(b)-\left.m_{\tilde{B}}(b)\right|^{m}\left[\mu\left(6^{k+1} B\right)\right]^{1-\frac{1}{p}}\right] \\
\leq & C\|b\|_{\mathrm{RBMO}(\mu)}^{m} M_{p, 30 \tau}(f)(x) \sum_{k=1}^{\infty}\left(k^{m}+1\right) \omega\left(6^{-k}\right) \frac{\mu\left(30 \tau \times 6^{k+1} B\right)}{\lambda\left(c_{B}, 6^{k} r_{B}\right)} \\
\leq & C M_{p, 30 \tau}(f)(x) .
\end{aligned}
$$

We have used the following fact in the last inequality:

$$
\int_{0}^{1} \frac{\omega(t)}{t}|\log t|^{m} d t \geq \sum_{k=1}^{\infty} \int_{6^{-k}}^{6^{1-k}} \frac{\omega\left(6^{-k}\right)}{6^{1-k}}\left|\log 6^{-k}\right|^{m} d t \geq C \sum_{k=1}^{\infty} k^{m} \omega\left(6^{-k}\right) .
$$

To estimate $B_{32}$, for all $y, w \in B$, if $\rho \in(0, \infty)$, by (1.9), Hölder's inequality and Lemma 2.4, we get

$$
\begin{aligned}
F_{32} \leq & C \int_{\mathcal{X} \backslash 6 B}\left|\frac{d(w, z)}{\lambda(w, d(w, z))}\right|\left|\frac{d(w, y)}{d(y, z)^{2-\rho}}\right|\left|b(z)-m_{\tilde{B}}(b)\right|^{m}|f(z)| \frac{1}{|d(y, z)|^{\rho}} d \mu(z) \\
\leq & C \sum_{k=1}^{\infty} 6^{-k} \frac{1}{\lambda\left(c_{B}, 6^{k} r_{B}\right)} \int_{6^{k+1} B \backslash 6^{k} B}\left|b(z)-m_{\tilde{B}}(b)\right|^{m}|f(z)| d \mu(z) \\
\leq & C \sum_{k=1}^{\infty} 6^{-k} \frac{1}{\lambda\left(c_{B}, 6^{k} r_{B}\right)}\left[\int_{6^{k+1} B}\left|b(z)-m_{6^{k+1} B}(b)\right|^{m}|f(z)| d \mu(z)\right. \\
& \left.+\int_{6^{k+1} B}\left|m_{6^{k+1} B}(b)-m_{\tilde{B}}(b)\right|^{m}|f(z)| d \mu(z)\right] \\
\leq & C \sum_{k=1}^{\infty} 6^{-k} \frac{1}{\lambda\left(c_{B}, 6^{k} r_{B}\right)}\left(\int_{6^{k+1} B}|f(z)|^{p} d \mu(z)\right)^{\frac{1}{p}} \\
& \times\left[\left(\int_{6^{k+1} B}\left|b(z)-m_{6^{k+1} B}(b)\right|^{m \cdot p^{\prime}} d \mu(z)\right)^{\frac{1}{p^{\prime}}}\right.
\end{aligned}
$$




$$
\begin{aligned}
& \left.+k^{m}\|b\|_{\mathrm{RBMO}(\mu)}^{m}\left[\mu\left(6^{k+1} B\right)\right]^{1-\frac{1}{p}}\right] \\
\leq & C\|b\|_{\mathrm{RBMO}(\mu)}^{m} M_{p, 30 \tau}(f)(x) \sum_{k=1}^{\infty} \frac{k^{m}+1}{6^{k}} \frac{\mu\left(30 \tau \times 6^{k+1} B\right)}{\lambda\left(c_{B}, 6^{k} r_{B}\right)} \\
\leq & C M_{p, 30 \tau}(f)(x) .
\end{aligned}
$$

Then we have

$$
F_{3} \leq C M_{p,(30 \tau)}(f)(x) .
$$

Moreover, combining the estimates of $E_{1}, E_{2}, F_{1}, F_{2}$, and $F_{3}$, we obtain the desired inequality (2.9).

Next we give the proof of (2.10). Write

$$
\begin{aligned}
& \left|h_{B}-h_{S}\right| \\
& =\left|m_{B}\left(\mathcal{M}^{\rho}\left(\left[b-m_{\tilde{B}}(b)\right]^{m} f \chi \mathcal{X} \backslash 6 B\right)\right)-m_{S}\left(\mathcal{M}^{\rho}\left(\left[b-m_{S}(b)\right]^{m} f \chi \mathcal{X} \backslash 6 S\right)\right)\right| \\
& \leq\left|m_{B}\left(\mathcal{M}^{\rho}\left(\left[b-m_{\tilde{B}}(b)\right]^{m} f \chi_{\mathcal{X} \backslash 6^{N_{1}}}\right)\right)-m_{S}\left(\mathcal{M}^{\rho}\left(\left[b-m_{\tilde{B}}(b)\right]^{m} f \chi_{\mathcal{X} \backslash 6^{N_{1} B}}\right)\right)\right| \\
& +\left|m_{S}\left(\mathcal{M}^{\rho}\left(\left[b-m_{\tilde{B}}(b)\right]^{m} f \chi_{\mathcal{X} \backslash 6^{N_{1}}}\right)\right)-m_{S}\left(\mathcal{M}^{\rho}\left(\left[b-m_{S}(b)\right]^{m} f \chi_{\mathcal{X} \backslash 6^{N_{1} B}}\right)\right)\right| \\
& +\left|m_{B}\left(\mathcal{M}^{\rho}\left(\left[b-m_{\tilde{B}}(b)\right]^{m} f \chi_{6^{N_{1}}}{ }_{B \backslash 6 B}\right)\right)\right|+\left|m_{S}\left(\mathcal{M}^{\rho}\left(\left[b-m_{S}(b)\right]^{m} f \chi_{6^{N_{1}}}{ }_{B \backslash 6 S}\right)\right)\right| \\
& :=G_{1}+G_{2}+G_{3}+G_{4} \text {. }
\end{aligned}
$$

To estimate $G_{1}$, it being similar to $E_{3}$, we get

$$
G_{1} \leq C M_{p, 30 \tau}(f)(x) .
$$

For $G_{2}$, we use

$$
\begin{aligned}
& {\left[b(z)-m_{\tilde{B}}(b)\right]^{m}-\left[b(z)-m_{S}(b)\right]^{m}} \\
& =\sum_{k=0}^{m-1} C_{m}^{k}\left[b(z)-m_{S}(b)\right]^{k} \cdot\left[m_{S}(b)-m_{\tilde{B}}(b)\right]^{m-k}, \\
& {\left[b(z)-m_{S}(b)\right]^{k}=\sum_{i=0}^{k} C_{k}^{i}[b(z)-b(y)]^{i} \cdot\left[b(y)-m_{S}(b)\right]^{k-i} .}
\end{aligned}
$$

So we have

$$
\begin{aligned}
& \left|\mathcal{M}^{\rho}\left(\left[b-m_{\tilde{B}}(b)\right]^{m} f \chi_{\mathcal{X} \backslash 6^{N_{1} B}}\right)-\mathcal{M}^{\rho}\left(\left[b-m_{S}(b)\right]^{m} f \chi_{\mathcal{X} \backslash 6^{N_{1} B}}\right)\right| \\
& \quad \leq\left|\mathcal{M}^{\rho}\left[\left(\left[b-m_{\tilde{B}}(b)\right]^{m}-\left[b-m_{S}(b)\right]^{m}\right) f \chi_{\mathcal{X} \backslash 6^{N_{1} B}}\right]\right| \\
& \quad \leq \sum_{k=0}^{m-1} C_{m}^{k}\left|m_{\tilde{B}}(b)-m_{S}(b)\right|^{m-k} \mathcal{M}^{\rho}\left(\left[b-m_{S}(b)\right]^{k} f \chi_{\mathcal{X} \backslash 6^{N_{1}}}\right)(y) \\
& \quad \leq C \sum_{k=0}^{m-1}\left[K_{B, S}\right]^{m-k} \mathcal{M}^{\rho}\left(\left[b-m_{S}(b)\right]^{k} f \chi_{\mathcal{X} \backslash 6^{N_{1}}}\right)(y)
\end{aligned}
$$




$$
\begin{aligned}
\leq & C \sum_{k=0}^{m-1}\left[K_{B, S}\right]^{m-k}\left[\sum_{i=0}^{k} C_{k}^{i}\left|m_{S}(b)-b(y)\right|^{k-i} \mathcal{M}_{b, i}^{\rho}\left(f \chi_{\mathcal{X} \backslash 6^{N_{1} B}}\right)(y)\right] \\
\leq & C \sum_{k=0}^{m-1}\left[K_{B, S}\right]^{m-k}\left(\sum_{i=0}^{k} C_{k}^{i}\left|m_{S}(b)-b(y)\right|^{k-i} \mathcal{M}_{b, i}^{\rho}(f)(y)\right) \\
& +C \sum_{k=0}^{m-1}\left[K_{B, S}\right]^{m-k} \mathcal{M}^{\rho}\left(\left|b-m_{S}(b)\right|^{k} f \chi_{6^{N_{1}}}(6) b B\right)(y) \\
& +C \sum_{k=0}^{m-1}\left[K_{B, S}\right]^{m-k} \mathcal{M}^{\rho}\left(\left|b-m_{S}(b)\right|^{k} f \chi_{6 B}\right)(y) .
\end{aligned}
$$

Therefore,

$$
\begin{aligned}
G_{2}= & m_{S}\left[C \sum_{k=0}^{m-1}\left[K_{B, S}\right]^{m-k}\left(\sum_{i=0}^{k}\left|m_{S}(b)-b(y)\right|^{k-i} M_{b, i}^{\rho}(f)\right)\right] \\
& +m_{S}\left[C \sum_{k=0}^{m-1}\left[K_{B, S}\right]^{m-k} \mathcal{M}^{\rho}\left(\left[b-m_{S}(b)\right]^{k} f \chi_{6^{N_{1}} B \backslash 6 B}\right)\right] \\
& +m_{S}\left[C \sum_{k=0}^{m-1}\left[K_{B, S}\right]^{m-k} \mathcal{M}^{\rho}\left(\left[b-m_{S}(b)\right]^{k} f \chi_{6 B}\right)\right] \\
:= & H_{1}+H_{2}+H_{3} .
\end{aligned}
$$

With the same argument as for $E_{1}$, we get

$$
H_{1} \leq C\left[K_{B, S}\right]^{m} \sum_{k=0}^{m-1} M_{\eta, 30 \tau}\left(M_{b, k}^{\rho}(f)\right)(x) .
$$

The estimates of $H_{2}$ and $H_{3}$ is very similar to $G_{4}$ and $E_{2}$, respectively, then we have

$$
\begin{aligned}
& H_{2} \leq C\left[K_{B, S}\right]^{m} M_{p, 30 \tau}(f)(x), \\
& H_{3} \leq C\left[K_{B, S}\right]^{m} M_{p, 30 \tau}(f)(x) .
\end{aligned}
$$

Therefore, combining $H_{1}, H_{2}, H_{3}$, we have

$$
G_{2} \leq C\left[K_{B, S}\right]^{m}\left[\sum_{k=0}^{m-1} M_{\eta, 30 \tau}\left(\mathcal{M}_{b, k}^{\rho}(f)\right)(x)+M_{p, 30 \tau}(f)(x)\right] .
$$

For $G_{3}$, by (1.9), the Minkowski inequality, Hölder's inequality, we obtain

$$
\begin{aligned}
& \left|\mathcal{M}^{\rho}\left(\left[b-m_{\tilde{B}}(b)\right]^{m} f_{6^{N_{1}}}{ }_{B \backslash 6 B}\right)\right| \\
& =\left(\int_{0}^{\infty}\left|\frac{1}{t^{\rho}} \int_{d(y, t)<t} \frac{K(y, z)}{|d(y, z)|^{1-\rho}}\left[b(z)-m_{\tilde{B}}(b)\right]^{m} f \chi_{6^{N_{1}} B \backslash 6 B} d \mu(z)\right|^{2} \frac{d t}{t}\right)^{\frac{1}{2}} \\
& \leq \int_{6^{N_{1}} B \backslash 6 B} \frac{|d(y, z)|^{\rho}}{\lambda(y, d(y, z))}\left|b(z)-m_{\tilde{B}}(b)\right|^{m}|f(z)| \frac{1}{|d(y, z)|^{\rho}} d \mu(z)
\end{aligned}
$$




$$
\begin{aligned}
\leq & C \sum_{k=1}^{N_{1}-1} \int_{6^{k+1} B \backslash 6^{k} B} \frac{1}{\lambda\left(c_{B}, 6^{k} r_{B}\right)}\left|b(z)-m_{\tilde{B}}(b)\right|^{m}|f(z)| d \mu(z) \\
\leq & C \sum_{k=1}^{N_{1}-1} \frac{1}{\lambda\left(c_{B}, 6^{k} r_{B}\right)}\left[\int_{6^{k+1} B}\left|b(z)-m_{6^{k+1} B}(b)\right|^{m}|f(z)| d \mu(z)\right. \\
& \left.+\int_{6^{k+1} B}\left|m_{6^{k+1} B}(b)-m_{\tilde{B}}(b)\right|^{m}|f(z)| d \mu(z)\right] \\
\leq & C \sum_{k=1}^{N_{1}-1} \frac{1}{\lambda\left(c_{B}, 6^{k} r_{B}\right)}\left(\int_{6^{k+1} B}|f(z)|^{p} d \mu(z)\right)^{\frac{1}{p}} \\
& \times\left[\left(\int_{6^{k+1} B}\left|b(z)-m_{6^{k+1} B}(b)\right|^{m \cdot p^{\prime}} d \mu(z)\right)^{\frac{1}{p^{\prime}}}\right. \\
& \left.+k^{m}\|b\|_{\mathrm{RBMO}(\mu)}^{m}\left[\mu\left(30 \tau \times 6^{k+1} B\right)\right]^{1-\frac{1}{p}}\right] \\
\leq & C\|b\|_{\mathrm{RBMO}(\mu)}^{m} M_{p, 30 \tau}(f)(x) \sum_{k=1}^{N_{1}-1}\left[\left(k^{m}+1\right) \frac{\mu\left(30 \tau \times 6^{k+1} B\right)}{\lambda\left(c_{B}, 6^{k} r_{B}\right)}\right] \\
\leq & C M_{p, 30 \tau}(f)(x) .
\end{aligned}
$$

Therefore,

$$
G_{3} \leq C M_{p, 30 \tau}(f)(x) .
$$

Similarly,

$$
G_{4} \leq C M_{p, 30 \tau}(f)(x) .
$$

Since (2.10) has been proved, Lemma 2.6 follows directly.

By Lemma 2.5 and the Marcinkiewicz interpolation theorem, we have

$$
\left\|\mathcal{M}^{\rho}(f)\right\|_{L^{p}(\mu)} \leq C\|f\|_{L^{p}(\mu)}
$$

Then using (2.3), (2.4), Lemma 2.6 and Lemma 2.1, we get

$$
\begin{aligned}
& \left\|\mathcal{M}_{b}^{\rho}(f)\right\|_{L^{p}(\mu)} \\
& \quad \leq\left\|N_{\delta}\left(\mathcal{M}_{b}^{\rho}(f)\right)\right\|_{L^{p}(\mu)} \\
& \quad \leq\left\|M_{\delta}^{\sharp}\left(\mathcal{M}_{b}^{\rho}(f)\right)\right\|_{L^{p}(\mu)} \\
& \quad \leq C\|b\|_{\mathrm{RBMO}(\mu)}\left\|\left[M_{\eta, 30 \tau}\left(\mathcal{M}^{\rho}(f)\right)(x)+M_{p, 30 \tau}(f)(x)\right]\right\|_{L^{p}(\mu)} \\
& \quad \leq C\|b\|_{\mathrm{RBMO}(\mu)}\|f\|_{L^{p}(\mu)} .
\end{aligned}
$$

We set

$$
\left\|\mathcal{M}_{b, m-1}^{\rho}(f)\right\|_{L^{p}(\mu)} \leq C\|b\|_{\mathrm{RBMO}}^{m-1}\|f\|_{L^{p}(\mu)} .
$$


Finally, using mathematical induction, (2.3), (2.4), Lemma 2.6 and Lemma 2.1, we obtain

$$
\begin{aligned}
& \left\|\mathcal{M}_{b, m}^{\rho}(f)\right\|_{L^{p}(\mu)} \\
& \quad \leq\left\|N_{\delta}\left(\mathcal{M}_{b, m}^{\rho}(f)\right)\right\|_{L^{p}(\mu)} \leq\left\|M_{\delta}^{\sharp}\left(\mathcal{M}_{b, m}^{\rho}(f)\right)\right\|_{L^{p}(\mu)} \\
& \quad \leq C\left[\sum_{k=0}^{m-1}\|b\|_{\mathrm{RBMO}(\mu)}^{m-k}\left\|M_{\eta, 30 \tau}\left(\mathcal{M}_{b, k}^{\rho}(f)\right)\right\|_{L^{p}(\mu)}+\|b\|_{\mathrm{RBMO}(\mu)}^{m}\left\|M_{p, 30 \tau}(f)\right\|_{L^{p}(\mu)}\right] . \\
& \quad \leq C\|b\|_{\mathrm{RBMO}}^{m}\|f\|_{L^{p}(\mu)} .
\end{aligned}
$$

Then Theorem 1.2 is proved.

\section{Proof of Theorem 1.3}

In this section, we will prove Theorem 1.3. We recall the boundedness in Morrey space $M_{p}^{q}(\mu)$ of the sharp maximal function on $(\mathcal{X}, d, \mu)[22,31]$.

Lemma 3.1 Let $f \in L_{\text {loc }}^{1}(\mu)$ satisfy $\int_{\mathcal{X}} f(x) d \mu(x)=0$ when $\|\mu\|:=\mu(\mathcal{X})<\infty$. Let $1<p \leq$ $q<\infty, \delta \in(0,1)$. If $\inf \left\{1, N_{\delta} f\right\} \in M_{p}^{q}(\mu)$, then there exists a constant $C>0$, such that

$$
\left\|N_{\delta} f\right\|_{M_{p}^{q}(\mu)} \leq C\left\|M_{\delta}^{\sharp}(f)\right\|_{M_{p}^{q}(\mu)} .
$$

Lemma 3.2 Let $\zeta>1,1<s<p \leq q<\infty, M_{s, \zeta} f$ be as in (2.2) is bounded on Morrey space $M_{p}^{q}(\mu)$, that is,

$$
\left\|M_{s, \zeta} f\right\|_{M_{p}^{q}(\mu)} \leq C\|f\|_{M_{p}^{q}(\mu)} .
$$

Next we give the proof of Theorem 1.3.

In combination with Lemma 2.6, the differences between the proof of Theorem 1.2 and Theorem 1.3 are as follows:

By (1.7) and (2.12), it is easy to see

$$
\begin{aligned}
\left\|\mathcal{M}^{\rho}(f)\right\|_{M_{p}^{q}(\mu)} & =\sup _{B} \mu(\kappa B)^{\frac{1}{q}-\frac{1}{p}}\left(\int_{B}\left|\mathcal{M}^{\rho}(f)\right|^{p} d \mu\right)^{\frac{1}{p}} \\
& =\sup _{B} \mu(\kappa B)^{\frac{1}{q}-\frac{1}{p}}\left\|\mathcal{M}^{\rho}(f)\right\|_{L^{q}(\mu)} \\
& \leq C \sup _{B} \mu(\kappa B)^{\frac{1}{q}-\frac{1}{p}}\|f\|_{L^{q}(\mu)} \\
& \leq C\|f\|_{M_{p}^{q}(\mu)} .
\end{aligned}
$$

Then using (2.3), (3.1), Lemma 2.6 and (2.2), we have

$$
\begin{aligned}
\left\|\mathcal{M}_{b}^{\rho}(f)\right\|_{M_{p}^{q}(\mu)} & \leq\left\|N_{\delta}\left(\mathcal{M}_{b}^{\rho}(f)\right)\right\|_{M_{p}^{q}(\mu)} \leq\left\|M_{\delta}^{\sharp}\left(\mathcal{M}_{b}^{\rho}(f)\right)\right\|_{M_{p}^{q}(\mu)} \\
& \leq C\|b\|_{\operatorname{RBMO}(\mu)}\left(\left\|M_{\eta, 30 \tau}\left(\mathcal{M}^{\rho}(f)\right)\right\|_{M_{p}^{q}(\mu)}+\left\|M_{p, 30 \tau}(f)\right\|_{M_{p}^{q}(\mu)}\right) \\
& \leq C\|b\|_{\operatorname{RBMO}(\mu)}\|f\|_{M_{p}^{q}(\mu)} .
\end{aligned}
$$


We set

$$
\left\|\mathcal{M}_{b, m-1}^{\rho}(f)\right\|_{M_{p}^{q}(\mu)} \leq C\|b\|_{\mathrm{RBMO}}^{m-1}\|f\|_{M_{p}^{q}(\mu)} .
$$

Finally, by mathematical induction, (2.3), (3.1), Lemma 2.6 and (2.2), we get

$$
\begin{aligned}
& \left\|\mathcal{M}_{b, m}^{\rho}(f)\right\|_{M_{p}^{q}(\mu)} \\
& \quad \leq\left\|N_{\delta}\left(\mathcal{M}_{b, m}^{\rho}(f)\right)\right\|_{M_{p}^{q}(\mu)} \leq\left\|M_{\delta}^{\sharp}\left(\mathcal{M}_{b, m}^{\rho}(f)\right)\right\|_{M_{p}^{q}(\mu)} \\
& \leq C\left[\sum_{k=0}^{m-1}\|b\|_{\mathrm{RBMO}(\mu)}^{m-k}\left\|M_{\eta, 30 \tau}\left(\mathcal{M}_{b, k}^{\rho}(f)\right)\right\|_{M_{p}^{q}(\mu)}+\|b\|_{\mathrm{RBMO}(\mu)}^{m}\left\|M_{p, 30 \tau}(f)\right\|_{M_{p}^{q}(\mu)}\right] . \\
& \quad \leq C\|b\|_{\mathrm{RBMO}(\mu)}^{m}\|f\|_{M_{p}^{q}(\mu)} .
\end{aligned}
$$

So, the proof of Theorem 1.3 is finished.

\section{Proof of Corollary 1.4}

If $\rho=1, m=1$ on Corollary 1.4, which is Theorem 1.10 of [26]. The different between Corollary 1.4 and Theorem 1.10 of [26] is to estimate $F_{31}$. So, in order to complete the proof of Corollary 1.4, it suffices to show that

$$
\begin{aligned}
F_{31} \leq & \|f\|_{L^{\infty}(\mu)} \sum_{k=1}^{\infty} \int_{6^{k+1} B \backslash 6^{k} B} \frac{|K(y, z)-K(w, z)|}{d(y, z)}\left|b(z)-m_{\tilde{B}}(b)\right|^{m} d \mu(z) \\
\leq & \|f\|_{L^{\infty}(\mu)} \sum_{k=1}^{\infty} \int_{6^{k+1} B \backslash 6^{k} B} \frac{|K(y, z)-K(w, z)|}{d(y, z)}\left|b(z)-m_{6^{k+1} B}(b)\right|^{m} d \mu(z) \\
& +\|f\|_{L^{\infty}(\mu)} \sum_{k=1}^{\infty}\left|m_{6^{k+1} B}(b)-m_{\tilde{B}}(b)\right|^{m} \int_{6^{k+1} B \backslash 6^{k} B} \frac{|K(y, z)-K(w, z)|}{d(y, z)} d \mu(z) \\
=: & F_{31}^{1}+F_{31}^{2} .
\end{aligned}
$$

Using Lemma 2.3, Lemma 2.4 in [26], (1.9) and (1.16), we have

$$
\begin{aligned}
F_{31}^{1} \leq & \|f\|_{L^{\infty}(\mu)} \sum_{k=1}^{\infty} \int_{6^{k+1} B \backslash 6^{k} B} \frac{|K(y, z)-K(w, z)|}{d(y, z)} \\
& \times \log ^{m}\left[2+6^{k} \cdot \mu\left(30 \tau \times 6^{k} B\right) \frac{|K(y, z)-K(w, z)|}{d(y, z)}\right] d \mu(z) \\
& +\|f\|_{L^{\infty}(\mu)} \sum_{k=1}^{\infty} \frac{1}{6^{k} \cdot \mu\left(30 \tau \times 6^{k} B\right)} \int_{6^{k+1} B} \exp \left(\left|b(z)-m_{6^{k+1} B}(b)\right|\right) d \mu(z) \\
\leq & \|f\|_{L^{\infty}(\mu)}+\|f\|_{L^{\infty}(\mu)} \sum_{k=1}^{\infty} k^{m} \int_{6^{k+1} B \backslash 6^{k} B} \frac{|K(y, z)-K(w, z)|}{d(y, z)} \\
& \times \log ^{m}\left(2+\frac{\mu\left(30 \tau \times 6^{k} B\right)}{\lambda\left(c_{B}, d(y, z)\right)}\right) d \mu(z) \\
\leq & \|f\|_{L^{\infty}(\mu)}
\end{aligned}
$$


and

$$
\begin{aligned}
F_{31}^{2} & \leq\|f\|_{L^{\infty}(\mu)} \sum_{k=1}^{\infty} k^{m} \int_{6^{k+1} B \backslash 6^{k} B}|K(y, z)-K(w, z)| \frac{1}{d(y, z)} d \mu(z) \\
& \leq\|f\|_{L^{\infty}(\mu)} .
\end{aligned}
$$

This completes the proof of Corollary 1.4.

Using the similar to the argument in the proof of Corollary 1.4, we can get Corollary 1.5.

\section{Some applications}

Now we give the applications of Theorem 1.2 and Theorem 1.3 for the classical parametric Marcinkiewicz integral.

Let $\Omega$ be homogeneous of degree zero in $R^{d}$ for $d \geq 2$, integrable and have mean value zero on the unit sphere $S^{d-1}$. In addition, $\Omega$ satisfies the following condition: with a constant $C>0$, for $x, x^{\prime}, y \in R^{d}$ and $\left|x-x^{\prime}\right| \leq \frac{|x-y|}{2}$,

$$
\left|\Omega(x-y)-\Omega\left(x^{\prime}-y\right)\right| \leq C \omega\left(\frac{\left|x-x^{\prime}\right|}{|x-y|}\right)
$$

where $\omega$ satisfies (1.24).

$\mu_{\Omega}^{\rho}$ be as in (1.1), where $\Omega$ satisfies the above condition (5.1). Moreover, $\mu_{\Omega, b, m}^{\rho}$ is generated by $\mu_{\Omega}^{\rho}$ with RBMO functions $b$, defined by

$$
\mu_{\Omega, b, m}^{\rho}(f)(x)=\left(\int_{0}^{\infty}\left|\frac{1}{t^{\rho}} \int_{|x-y|<t} \frac{\Omega(x-y)}{|x-y|^{d-\rho}}[b(x)-b(y)]^{m} f(y) d y\right|^{2} \frac{d t}{t}\right)^{\frac{1}{2}}
$$

where $0<\rho<d$.

Theorem 5.1 Let $0<\rho<d$ and $\Omega$ satisfies (5.1). $\mu_{\Omega, b, m}^{\rho}(f)$ be as in (5.2), and $\omega$ satisfies (1.24), then there exists a constant $C>0$, for all $f \in L^{p}\left(R^{d}\right), 1<p<\infty$ such that

$$
\left\|\mu_{\Omega, b, m}^{\rho}(f)\right\|_{L^{p}\left(R^{d}\right)} \leq C\|b\|_{\mathrm{RBMO}\left(R^{d}\right)}^{m}\|f\|_{L^{p}\left(R^{d}\right)}
$$

For all $f \in M_{p}^{q}\left(R^{d}\right), 1<p \leq q<\infty$, such that

$$
\left\|\mu_{\Omega, b, m}^{\rho}(f)\right\|_{M_{p}^{q}\left(R^{d}\right)} \leq C\|b\|_{\mathrm{RBMO}\left(R^{d}\right)}^{m}\|f\|_{M_{p}^{q}\left(R^{d}\right)} .
$$

Next, we give the applications of Theorem 1.2 and Theorem 1.3 for the parametric Marcinkiewicz integral operator in Euclidean space where $\mu$ satisfies the growth condition (1.2).

Let $\omega$ satisfy (1.24), $K$ satisfy (1.17) and the following conditions hold with a constant $C>0$ :

(b') $\left|K(x, y)-K\left(x^{\prime}, y\right)\right| \leq C \frac{1}{|x-y|^{d-1}} \omega\left(\frac{\left|x-x^{\prime}\right|}{|x-y|}\right)$, 
where $x, x^{\prime}, y \in R^{d}$ and $\left|x-x^{\prime}\right| \leq \frac{|x-y|}{2}$.

$$
\text { (c') }\left|K(x, y)-K\left(x, y^{\prime}\right)\right| \leq C \frac{1}{|x-y|^{d-1}} \omega\left(\frac{\left|y-y^{\prime}\right|}{|x-y|}\right),
$$

where $x, y^{\prime}, y \in R^{d}$ and $\left|y-y^{\prime}\right| \leq \frac{|x-y|}{2}$.

Define the parametric Marcinkiewicz integral operator $M^{\rho}$ with respect to the kernel above as follows:

$$
M^{\rho}(f)(x)=\left(\int_{0}^{\infty}\left|\frac{1}{t^{\rho}} \int_{|x-y|<t} \frac{K(x, y)}{|x-y|^{1-\rho}} f(y) d \mu(y)\right|^{2} \frac{d t}{t}\right)^{\frac{1}{2}}, \quad 0<\rho<\infty
$$

$M_{b, m}^{\rho}$ is generated by $M^{\rho}$ with RBMO functions $b$, defined by

$$
M_{b, m}^{\rho}(f)(x)=\left(\int_{0}^{\infty}\left|\frac{1}{t^{\rho}} \int_{|x-y|<t} \frac{K(x, y)}{|x-y|^{1-\rho}}[b(x)-b(y)]^{m} f(y) d \mu(y)\right|^{2} \frac{d t}{t}\right)^{\frac{1}{2}},
$$

Theorem 5.2 Let $0<\rho<\infty$, and $K$ satisfythe above conditions (1.17), ( $\left.\mathrm{b}^{\prime}\right)$ and $\left(\mathrm{c}^{\prime}\right)$. Let $M^{\rho}, M_{b, m}^{\rho}$ be as in (5.3) and (5.4). Suppose that $M^{\rho}$ is bounded on $L^{2}(\mu), b \in \operatorname{RBMO}(\mu), \omega$ satisfies (1.24), then there exists a constant $C>0$, for all $f \in L^{p}(\mu), 1<p<\infty$ such that

$$
\left\|M_{b, m}^{\rho}(f)\right\|_{L^{p}(\mu)} \leq C\|b\|_{\mathrm{RBMO}(\mu)}^{m}\|f\|_{L^{p}(\mu)} .
$$

For all $f \in M_{p}^{q}\left(R^{d}\right), 1<p \leq q<\infty$, we have

$$
\left\|M_{b, m}^{\rho}(f)\right\|_{M_{p}^{q}(\mu)} \leq C\|b\|_{\mathrm{RBMO}(\mu)}^{m}\|f\|_{M_{p}^{q}(\mu)} .
$$

\section{Acknowledgements}

The authors thank the anonymous referee for reading the paper carefully and giving some useful advices.

\section{Funding}

This work was supported partially by the NNSF of China (No. 11771399, 11961056) and Zhejiang University of Science and Technology graduate research innovation fund.

Availability of data and materials

Not applicable.

\section{Competing interests}

The authors declare that they have no competing interests.

Authors' contributions

All authors read and approved the final manuscript.

\section{Publisher's Note}

Springer Nature remains neutral with regard to jurisdictional claims in published maps and institutional affiliations.

Received: 3 January 2021 Accepted: 22 June 2021 Published online: 02 July 2021

\section{References}

1. Hörmander, L.: Estimates for translation invariant operators in $\mathrm{L}^{\mathrm{p}}$ spaces. Math. Res. Lett. 104, 93-104 (1960)

2. Fan, D.S., Sato, S.C.: Weak type $(1,1)$ estimates for Marcinkiewicz integrals with rough kernels. Tohoku Math. J. 53 , 265-284 (2001)

3. Stein, E.M.: On the functions of Littlewood-Paley, Lusin, and Marcinkiewicz. Trans. Am. Math. Soc. 88, 430-466 (1958)

4. Torchinsky, A., Wang, S.L.: A note on the Marcinkiewicz integral. Colloq. Math. 60, 235-243 (1990)

5. Al-Salman, A., Al-Qassem, C.L.C.H., Pan, Y.: $\mathrm{L}^{p}$ bounds for the function of Marcinkiewicz. Math. Res. Lett. 9, 697-700 (2002) 
6. Gürbüz, F.: Generalized local Morrey spaces and multilinear commutators generated by Marcinkiewicz integrals with rough kernel associated with Schrödinger operators and local Campanato functions. J. Appl. Anal. Comput. 8, 1369-1384 (2018)

7. Gürbüz, F.: Marcinkiewicz integrals with rough kernel associated with Schrödinger operators and commutators on generalized vanishing local Morrey spaces. Tbil. Math. J. 11, 133-156 (2018)

8. Gürbüz, F.: Generalized weighted Morrey estimates for Marcinkiewicz integrals with rough kernel associated with Schrödinger operator and their commutators. Chin. Ann. Math., Ser. B 41, 77-98 (2020)

9. Gürbüz, F.: Local Campanato estimates for multilinear commutator operators with rough kernel on generalized local Morrey spaces. J. Coupled Syst. Multiscale Dyn. 6, 71-79 (2018)

10. Gürbüz, F.: Ağırlıklı Campanato uzayları ve bu uzaylar üzerinde bazı eşitsizlikler. In: Cüneyt Ersanli, C. (ed.) Geleceğin Dünyasinda Bilimsel Ve Mesleki Çalişmalar 2019 Matematik Ve Fen Bilimleri, 1nd edn., pp. 52-66. Ekin Yayınevi, Turkish (2019)

11. Gürbüz, F: A note concerning Marcinkiewicz integral with rough kernel. Chin. Ann. Math., Ser. B 24, 1-14 (2021)

12. Gürbüz, F:: Genelleştirilmiş agıılıkıı Morrey uzaylarında değişken çekirdekli marcinkiewicz integralinin komütatörünün sınırlıı̆ı. In: Akdura, N. (ed.) Matematik Ve Fen Alaninda Uluslararasi Araştirmalar, 1nd edn., pp. 19-33. Eğitim Yayınevi, Turkish (2021)

13. Tao, X.X., Yu, X., Zhang, S.Y.: Boundedness on Hardy-Sobolev spaces for hypersingular Marcinkiewicz integrals with variable kernels. J. Inequal. Appl. 2008, 835938 (2008)

14. Lin, C.C., Lin, Y.C., Tao, X.X., Yu, X.: The boundedness of Marcinkiewicz integral with variable kernel. III. J. Math. 53, $197-217(2009)$

15. Tao, X.X., Hu, G.E.: A sparse domination for the Marcinkiewicz integral with rough kernel and applications. Publ. Math. (Debr.) 96, 377-399 (2020)

16. Tolsa, X.: Painlevés problem and the semiadditivity of analytic capacity. Acta Math. 190, 105-149 (2003)

17. Hytönen, T: A framework for non-homogeneous analysis on metric spaces, and the RBMO space of Tolsa. Publ. Mat. $54,485-504$ (2010)

18. Fu, X., Yang, D.C., Yuan, W.: Generalized fractional integrals and their commutators over non-homogeneous metric measure spaces. Taiwan. J. Math. 18, 509-557 (2014)

19. Zheng, T.T., Wang, Z., Xiao, W.L.: Maximal bilinear Calderón-Zygmund operators of type $\omega(t)$ on non-homogeneous space. Ann. Funct. Anal. 6, 134-154 (2015)

20. Zheng, T.T., Tao, X.X.: Boundedness for iterated commutators of multilinear singular integrals of Dini's type on non-homogeneous metric measure spaces. Sci. Sin., Math. 47, 1029-1046 (2017)

21. Bui, T.A., Duong, X.T.: Hardy spaces, regularized BMO spaces and the boundedness of Calderón-Zygmund operators on non-homogeneous spaces. J. Geom. Anal. 23, 895-932 (2013)

22. Cao, Y.G., Zhou, J.: Morrey spaces for non-homogeneous metric measure spaces. Abstr. Appl. Anal. 2013, Article ID 196459 (2013)

23. Tao, X.X., Sha, H.: The boundedness of multilinear operators on generalized Morrey spaces over the quasi-metric space of non-homogeneous type. J. Inequal. Appl. 2013, Article ID 330 (2013)

24. Lin, H.B., Yang, D.C.: Equivalent boundedness of Marcinkiewicz integrals on non-homogeneous metric measure spaces. Sci. China Math. 57, 123-144 (2014)

25. Fu, H.L., Lin, H.B., Meng, Y.: Boundedness of parametric Marcinkiewicz integral operators on non-homogeneous metric measure spaces. Sci. Sin., Math. 46, 1801-1814 (2016)

26. Cao, Y.G., Zhou, J.: The boundedness of Marcinkiewicz integrals commutators on non-homogeneous metric measure spaces. J. Inequal. Appl. 2015, Article ID 259 (2015)

27. Tao, S.P., Lu, G.H.: Commutators of Marcinkiewicz integrals with RBMO on Morrey spaces. J. Math. 62, 269-278 (2019)

28. Hu, G.E., Lin, H.B.: Marcinkiewicz integrals with non-doubling measures. Integral Equ. Oper. Theory 58, 205-238 (2007)

29. Zhang, J., Li, L.: The boundedness of Marcinkiewicz integral commutators on Morrey spaces with non-doubling measures. Inst. Appl. Math. 26, 131-137 (2010)

30. Fu, X., Yang, D.C., Yuan, W.: Boundedness of multilinear commutators of Calderón-Zygmund operators on Orlicz spaces over non-homogeneous spaces. Taiwan. J. Math. 16, 2203-2238 (2012)

31. Lu, G.H.: Commutator of bilinear $\theta$-type Calderón-Zygmund operators on Morrey spaces over non-homogeneous spaces. Anal. Math. 46, 97-118 (2020)

\section{Submit your manuscript to a SpringerOpen ${ }^{\circ}$ journal and benefit from:}

- Convenient online submission

- Rigorous peer review

- Open access: articles freely available online

- High visibility within the field

Retaining the copyright to your article

Submit your next manuscript at $\gg$ springeropen.com 